\title{
Elevated Carbohydrate Antigen 19-9 (CA 19-9) in Patients with Echinococcus Infection
}

\author{
Marc Pfister', Bruno Gottstein ${ }^{2}$, Robert Kretschmer ${ }^{3}$, \\ Thomas Cerny ${ }^{4}$ and Andreas Cerny ${ }^{1}$ \\ ${ }^{1}$ Department of Medicine, \\ ${ }^{2}$ Institute of Parasitology, \\ ${ }^{3}$ Department of Clinical Chemistry, \\ ${ }^{4}$ Institute of Oncology, \\ University of Bern, Switzerland
}

The carbohydrate antigen 19-9 (CA 19-9), a determinant (sialylated lacto-N-fucopentaose 119) of a circulating oligosaccharide antigen, is a frequently used tumor marker. Echinococcus spp. infects humans throughout the world and may be able to synthesize closely related molecules which could interfere with the measurement and interpretation of CA 19-9 concentration. The main objective of the present study was to determine the range of CA 19-9 levels in the sera of patients infected by E. granulosus (cystic hydatide disease; CYSHD) or $E$. multilocularis (alveolar hydatide disease; ALVHD).

Serum samples were collected from patients (aged 10-85 years) over a period of 5 years: from 19 patients with CYSHD and from 20 patients with ALVHD. Infection was confirmed by positive Echinococcus serology and clinical evidence provided by imaging and/or histopathological findings.

CA 19-9 was detectable in 13 patients with CYSHD $(13.5 \pm 8.5 \mathrm{kU} / \mathrm{I})$ and 13 patients with ALVHD (30.0 \pm 21 $k U / l ; p<0.05)$. Thus ALVHD patients exhibited a significantly higher plasma level of CA 19-9 than CYSHD patients. The serum level of CA 19-9 assessed with an increased cut-off value ( $>22 \mathrm{kU} / \mathrm{I}$ ) was elevated in nine $(45 \%)$ of 20 ALVHD patients compared to two $(11 \%)$ of 19 CYSHD patients $(p<0.05)$.

Sera from patients with Echinococcus multilocularis infection contain substances which cross-react with CA 19-9. These substances originate either from the parasite or are synthesized by the host in response to the infection, and possibly bear the Lewis-a antigen or closely related structures which are recognized by anti-CA 19-9 antibodies. Our findings are relevant to the investigation of patients presenting with cystic lesions for which the differential diagnosis includes an infectious or neoplastic origin.

Key words: Tumor markers; Carbohydrate antigen 19-9; Lacto-N-fucopentaose; Lewis-a antigen; Echinococcosis; Pancreatic infections.

Abbreviations: ALVHD, alveolar hydatide disease; AU, antibody units; CA 19-9, carbohydrate antigen; CYSHD, cystic hydatide disease; EgHF antigen, Echinococcus granulosus hydatide fluid antigen; Em2 antigen, Echinococcus multilocularis specific carbohydrate antigen; II/3-10 anti- gen, recombinant Echinococcus multilocularis antigen; spp, species.

\section{Introduction}

Echinococcus spp. infections in humans occur throughout the world (1). Cystic hydatide disease (CYSHD) is caused by E. granulosus. The liver is the most frequent site of hydatid cyst localization (> 65\%), followed by the lungs, spleen, kidneys, heart, bone and, rarely, the central nervous system. Alveolar hydatide disease (ALVHD) is caused by E. multilocularis, and the primary localization in humans is again the liver (> 95\%) (2). Hence a systemic infection with $E$. granulosus or E. multilocularis can mimic a metastatic cancer and should be taken into consideration as a differential diagnosis in malignant disease. As progressive ALVHD is one of the most lethal helminthic diseases in humans, early diagnosis of infection may considerably improve prognosis due to the potential for radical surgery. Only complete surgical removal of the entire parasite lesion offers prospects for curative treatment of the active infection (3). For patients without radical surgery, the mortality rate was found to be $92 \%$ within 10 years after primary diagnosis (4).

Carbohydrate antigen 19-9 (CA 19-9) is the carbohydrate determinant (sialylated lacto-N-fucopentaose 119) of a circulating oligosaccharide antigen which was detected originally, using a monoclonal antibody, in human colon carcinoma growing in cell culture. Raised serum values of the antigen have been found on glycoproteins in the sera of patients with colorectal, gastric, and pancreatic cancers (5). The normal range of CA 19-9 in serum of healthy subjects has been established in different studies, and generally a CA 19-9 level above $37 \mathrm{kU} / \mathrm{l}$ was found in less than $2 \%$ of the tested individuals (6-8).

The main objective of the present study was to determine the range of CA 19-9 concentrations in CYSHD and ALVHD patients. The idea stemmed from the present lack of data on CA 19-9 values in serum of such patients. The antigen may show serological cross-reactivity in that the parasite has been reported to synthesize closely related molecules (9-11). Such molecules may also potentially confuse with the oncological/prognostic interpretation of CA 19-9.

\section{Patients and Methods}

Serum samples were obtained from 19 patients with CYSHD and from 20 patients with ALVHD (20 females/19 males; median (SD) age $44 \pm 20$ years, range: 10 to 85 years). Infection 
was confirmed by positive Echinococcus serology complementing clinical evidence provided by imaging and/or histopathological findings. Sera were collected over a period of 5 years and kept frozen at $-80^{\circ} \mathrm{C}$ until use. In line with a previous study (12) documenting the diagnostic characteristics of the enzyme-linked immunosorbent assay (ELISA), sera were immunodiagnostically investigated using the $E$. granulosus hydatide fluid (EgHF-)antigen and the E. multilocularis carbohydrate (Em2) and the recombinant II/3-10 antigen. The test procedures were exactly as published earlier (12).

Samples from 19 patients with CYSHD and from 20 patients with ALVHD were thawed, mixed and centrifuged at $2000 \times g$ for 10 min prior to testing. CA 19-9 was determined using the automatic Boehringer Enzymun-Test ${ }^{\circledR}$ system ES300 (Boehringer Mannheim Diagnostics, Tutzing, Germany), according to the manufacturer's instructions. This ELISA is based on the 1116NS-119 antibody from Centocor (13). It uses streptavidin-coated tubes with a biotinylated first antibody and a peroxydase-labeled second antibody. The widely used cut-off concentration based on healthy subjects is $22.0 \mathrm{kU} / \mathrm{l}$ (95th percentile $=21.5 \mathrm{kU} / \mathrm{l})(13,14)$. In earlier studies, as mentioned above, the cut-off was set at $37 \mathrm{kU} / \mathrm{l}$ (99th percentile $=37 \mathrm{kU} / \mathrm{I})$.

Other manufacturers may indicate different reference ranges for CA 19-9, based either on the $99 \%$ distribution of the values in healthy subjects, or on cut-off values related to the diagnostic sensitivity for tumor detection. No cross-reactions between the antibodies used in the test and other antigens than CA 19-9 have been reported so far.

The statistics software package SYSTAT was used for data analysis (SYSTAT 6.01, Inc., Evanston, USA). Data are expressed as mean values \pm standard deviation (SD) Pearson Chi-square test $(2 \times 2$ tables $)$ and analysis of variance (ANOVA), including Tukey post hoc test, were applied as appropriate.

\section{Results}

The baseline immunodiagnostic results confirming Echinococcosis are documented in Table 1. ELISA for
EgHF was positive in 18 (95\%) of 19 patients with CYSHD and in 19 (95\%) of 20 patients with ALVHD, respectively. The E. multilocularis-specific Em2 and II/310 tests provided positive reactions in 16 and 8 cases respectively. When combining the results of both antigens, $19(95 \%)$ of the sera were positive in at least one of the tests.

The mean (SD) serum CA 19-9 level for all patients was $14.5 \pm 18 \mathrm{kU} / \mathrm{l}$. In patients with measurable levels of CA $19-9(\geq 0.05 \mathrm{kU} / \mathrm{l})$, serum values ranged from $5.1 \mathrm{kU} / \mathrm{l}$ to $74.2 \mathrm{kU} / \mathrm{l}$. The distribution of CA $19-9$ serum concentrations in CYSHD and ALVHD patients is shown in Figure 1.

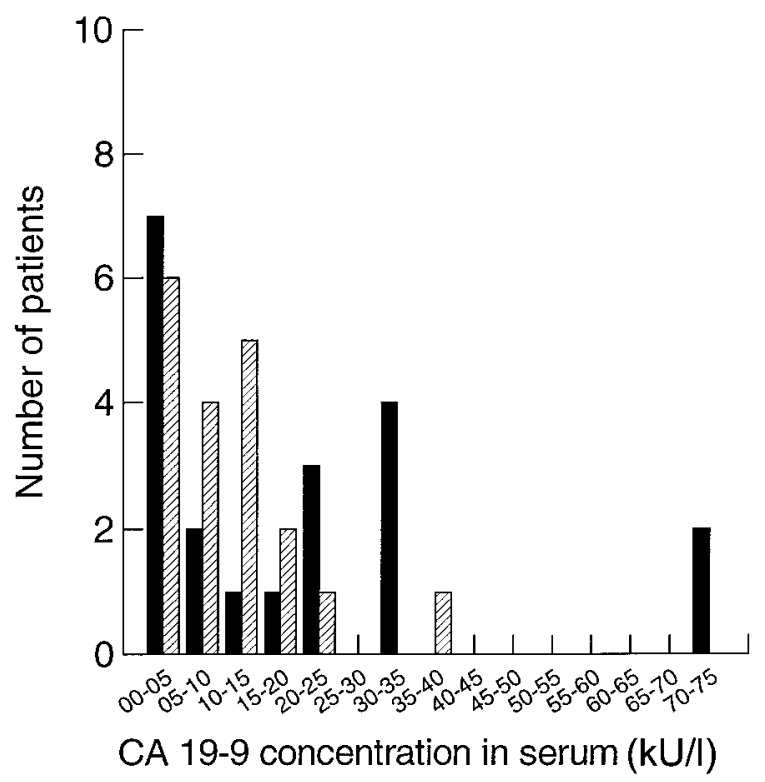

Fig. 1 Range and distribution of CA 19-9 serum concentrations in 19 patients with cystic hydatide disease (shaded bars) and in 20 patients with alveolar hydatide disease (black bars).
Tab. 1 Clinical and laboratory characteristics of patients with Echinococcus granulosus (cystic hydatide disease; CYSHD) or
Echinococcus multilocularis (alveolar hydatide disease; ALVHD) infection.

\begin{tabular}{|c|c|c|c|}
\hline & $\begin{array}{l}\text { Patients with } \\
\text { CYSHD }(n=19)\end{array}$ & $\begin{array}{l}\text { Patients with } \\
\text { ALVHD }(n=20)\end{array}$ & $\mathrm{p}$-Value \\
\hline Age (years; median, range) & $31(10$ to 80$)$ & 49 (32 to 85$)$ & $<0.05$ \\
\hline Female/male $(n)$ & $9 / 10$ & $11 / 9$ & ns \\
\hline Patients with CA $19-9<0.05 \mathrm{kU} / \mathrm{l}(\mathrm{n}, \%)$ & $6(32)$ & $7(35)$ & ns \\
\hline Patients with CA 19-9 0.05-22 kU/l (n, \%) & $11(58)$ & $4(20)$ & ns \\
\hline Patients with CA 19-9 > 22 kU/l (n, \%) & $2(11)$ & $9(45)$ & $<0.05$ \\
\hline CA 19-9 (kU/l) in patients with detectable & $13.5 \pm 8.5$ & $30.0 \pm 21$ & $<0.05$ \\
\hline \multicolumn{4}{|l|}{ CA $19-9(>0.05 \mathrm{kU} / \mathrm{l}),($ mean \pm SD) } \\
\hline EgHF-ELISA (AU; mean \pm SD) & $69.0 \pm 36$ & $71.2 \pm 34$ & ns \\
\hline Patients with positive EgHF-ELISA ( $n, \%$ ) & $18(95)$ & $19(95)$ & ns \\
\hline Patients with positive Em2-ELISA (n, \%) & $3(16)$ & $16(80)$ & $<0.01$ \\
\hline Patients with positive II/3-10-ELISA ( $n, \%$ ) & $0(0)$ & $8(40)$ & $<0.001$ \\
\hline
\end{tabular}

EgHF-ELISA: enzyme-linked immunosorbent assay (ELISA) for the E. granulosus hydatide fluid EgHF-antigen; Em2ELISA: ELISA for the E. multilocularis carbohydrate Em2; II/310-ELISA: ELISA for the recombinant II/3-10 antigen. AU de- notes antibody units. The carbohydrate antigen 19-9 (CA 19-9) was determined by using the automatic Boehringer EnzymunTest $^{\circledR}$ system ES300 based on the 1116NS-199 antibody from Centocor. ns: not significant. 
There was no correlation between serum values of CA $19.9(\mathrm{kU} / \mathrm{l})$ and the amount of antibody units (AU) of EgHF, Em2 and the recombinant antigen II/3-10. There was also no correlation with age of the patients

CA 19-9 was detectable in 13 patients with CYSHD and in 13 patients with ALVHD (Table 1). ALVHD patients exhibited higher serum levels of CA 19-9 than CYSHD patients $(13.5 \pm 8.5 \mathrm{kU} / \mathrm{l}$ vs. $30.0 \pm 21 \mathrm{kU} / \mathrm{l}, \mathrm{p}<$ 0.05). The serum level of CA $19-9$ assessed with higher cut-off value (> $22 \mathrm{kU} / \mathrm{l}$ ) was elevated in $9(45 \%)$ out of 20 ALVHD patients compared to $2(11 \%)$ out of 19 CYSHD patients $(p<0.05)$.

\section{Discussion}

The concentration of the tumor marker CA 19-9 was increased in $13(28 \%)$ of 39 sera from patients with CYSHD or ALVHD when assessed with a cut-off value based on healthy subjects (95th percentile: $22 \mathrm{kU} / \mathrm{l})(6$, $13,14)$. Del Villano et al. (6) showed that $99.6 \%$ of healthy blood donors have values below $37 \mathrm{kU} / \mathrm{l}$. However, for clinical screening purposes a cut-off value of $22 \mathrm{kU} / \mathrm{l}$ is commonly used, as opposed to the cut-off value of $37 \mathrm{kU} / \mathrm{l}$ (99th percentile).

The observation of increased CA 19-9 concentrations in patients with an Echinococcus infection is in line with previous reports of elevated serum CA 19-9 concentrations in patients with benign liver disease, including infectious diseases affecting the liver, such as viral hepatitis $(15,16)$. One of the main findings of our study was that the tumor marker CA 19-9 appeared more frequently, and was markedly elevated in sera from ALVHD patients as compared to CYSHD patients ( $45 \%$ vs. $11 \%$ ). A putative explanation for this could be provided by differences in the cellular and humoral immune responses to CYSHD and ALVHD (17-19). In ALVHD, the larval stage of E. multilocularis leads to an active and tumor-like proliferation of the primary infection in the liver. Conversely, ALVHD is not associated with containment of the lesions by reactive fibrosis. The involvement of cellular immunity in ALVHD leads to an intense granulomatous infiltration observed around the hepatic parasitic lesions $(17,18)$. A further emphasis on the tumor-like growth and behavior or $E$. multilocularis metacestode was given by the recent identification and characterization of the 14-3-3 protein overexpressed at the metacestode stage of the parasite (20). The 14-3-3 proteins seem to act as key molecules in the processes of cell differentiation and proliferation, and have also been directly implicated in abnormal cell proliferation phenomena $(21,22)$.

A large proportion of patients with ALVHD responds with a marked synthesis of parasite-specific antibodies, including all isotypes of immunoglobulins. However, humoral and cell-mediated immune responses are different in susceptible vs. resistant individuals (23). Since the course of cellular and humoral immune response is different in cured patients who had received radical surgery, compared to patients with only a partial surgery or no resection, we could postulate that the regulation of antigen synthesis, including "tumor-related" antigens such as the oligosaccharide CA 19-9, may also depend on the clinical course as well as on its relationship to surgical interventions and chemotherapy. This is underscored by our study where we included an additional follow-up of a 49-year-old male patient with hepatic CYSHD. This allowed us to study the time course of CA 19-9 serum concentrations after surgical removal of the parasitic lesion in the liver. We observed, subsequent to the radical resection of the hepatic cyst, a parallel decrease in CA 19-9 serum concentration and in EgHF-ELISA antibody level as compared to the preoperative values $(5.8 \mathrm{kU} / \mathrm{l}$ vs. 24.4 $\mathrm{kU} / \mathrm{l}$ and $0.0 \mathrm{AU}$ vs. $70 \mathrm{AU}$, respectively).

The epitope recognised by anti-CA 19-9 antibodies is the sialylated Lewis-a antigen (24), sialylated lacto-Nfucopentaose II. The Lewis-a antigen ends with a characteristic carbohydrate sequence (Gal $\beta$ 1-3 [Fuc $\alpha$ 1-4] Nac.). A glycosphingolipid with a very similar terminal residue has been found in metacestodes of E. multilocularis (Gal $\beta$ 1-6 [Fuc $\alpha$ 1-4] Gal.) (9), and other sialylated fucose-containing glycans were found in hydatid cyst membranes of E. granulosus (25).

Therefore, we postulate that the blood from patients with an Echinococcus infection may contain substances originating from the parasite which have been sialylated either by the host or by the parasite's metabolism. These substances may bear the Lewis-a antigen or closely related structures which are recognised by anti-CA 19-9 antibodies. Furthermore, it is conceivable that a subgroup of patients with ALVHD are able to produce an oligosaccharide similar to the CA 19-9 antigen, or to induce its synthesis by intrahepatic stimulation caused by the inflammation resulting from the E. multilocularis metacestode. The variability in the production of "tumor-related" antigens in different ALVHD patients could depend on their immunogenetic background. Putatively, other exogenous factors such as anthelmintic or surgical treatment may also be involved. These hypotheses need further investigation.

In conclusion, alveolar hydatide disease (E. multilocularis) in humans is usually a fatal disease when not diagnosed early enough to provide curative treatment such as radical surgery. Since $45 \%$ of the sera from patients with ALVHD demonstrate elevated CA 19-9 levels, we emphasize the need to consider an infection with E. multilocularis in patients with cystic lesions in the liver and/or in other organs, even if the tumor marker CA 19-9 ist elevated.

\section{References}

1. Gottstein B, Reichen J. Echinococcosis/Hydatidosis. In: Cook GC, editor. Manson's tropical diseases. London: Saunders Publishing, 1996:1486-58.

2. Schantz PM, Gottstein B. Echinococcosis (Hydatidosis). Immunodiagnosis of parasitic diseases. Academic Press 1986; 1:69-107.

3. WHO: Guidelines for treatment of cystic and alveolar echinococcosis in humans. WHO Informal Working 
Group on Echinococcosis. Bull World Health Organ 1996; 74:231-44.

4. Ammann RW, Tschudi K, von Ziegler M. Langzeitverlauf bei 60 Patienten mit alveolarer Echinokokkose unter Dauertherapie mit Mebendazol. Klin Wochenschr 1988; 66:1060-73.

5. Koprowski H, Herlyn M, Steplewski Z, Sears HF. Specific antigen in serum of patients with colon carcinoma. Science $1981 ; 212: 53-5$.

6. Del Villano BC, Bernnman S, Brock P, Bucher C, Liu V, McClure M. Radioimmunometric assay for a monoclonal antibody-defined tumor marker, CA 19-9. Clin Chem 1983; 29:549-52.

7. Yoshikawa T, Nishida K, Tanigawa M. Carbohydrate antigenic determinant (CA 19-9) and other tumor markers in gastrointestinal malignancies. Digestion 1985; 31:67-76.

8. Farini R, Fabris C, Bonvicini P. CA 19-9 in the differential diagnosis between pancreatic cancer and chronic pancreatitis. Eur J Cancer Clin Oncol 1985; 21:429-32.

9. Persat C, Couhours F. Glycosphingolipids with Gal $\beta$ 1-6 Gal sequences in metacestodes of the parasite Echinococcus multilocularis. J Biol Chem 1992; 267:8764-9.

10. Sato C, Furuya K. Isolation and characterization of a diagnostic polysaccharide antigen from larval Echinococcus multilocularis. Jpn J Med Sci Biol 1994; 47:65-71.

11. Ferreira AM, Wuerzner R, Hobart MJ, Lachmann PJ. Study of the in vitro activation of the complement alternative pathway by Echinococcus granulosus hydatid cyst fluid. Parasite Immunol 1995; 17:245-51.

12. Gottstein B, Jacquier P, Bersson-Hadni S, Eckert J. Improved primary immunodiagnosis of alveolar Echinococcosis in humans by an enzyme-linked immunosorbent assay using the EM2plus antigen. J Clin Microbiol 1993; 31:373-6.

13. Van Dalen A, Kessler A. A multicentre evaluation of tumour marker determinations using the automatic EnzymunTest ${ }^{\circledR}$ Systems ES 300 and ES 600/700. Eur J Clin Chem Clin Biochem 1996; 34:377-84.

14. 6th Symposium on Tumour Markers, Hamburg 1991. Tumour associated antigens, oncogenes, receptors, cytokines in tumour diagnosis and therapy at the beginning of the nineties. In: Klapdor R, editor. Munich: Zuckschwerdt Verlag, 1992:253-8.

15. Kew MC, Berger EL, Koprowski H. Carbohydrate antigen 19-9 as a serum marker of hepatocellular carcinoma: comparison with $\alpha$-fetoprotein. Br J Cancer 1987; 56:86-7.

16. Gupta MK, Arciaga R, Bocci L, Tubbs R, Bukowski R, Deod- har SD. Measurement of a monoclonal-antibody-defined antigen (CA 19-9) in the sera of patients with malignant and nonmalignant diseases. Cancer 1985; 56:277-83.

17. Bresson-Hadni S, Vuitton DA, Lenys D, Liance M, Racadot E, Miguet JP. Cellular immune response in Echinococcus multilocularis infection in humans. I. Lymphocyte reactivity to Echinococcus antigens in patients with alveolar echinococcosis. Clin Exp Immunol 1989; 78:61-6.

18. Bresson-Hadni S, Vuitton DA, Lenys D, Liance M, Racadot E, Miguet JP. Cellular immunity in experimental Echinococcus multilocularis infection. II. Sequential and comparative phenotypic study of the periparasitic mononunclear cells in resistant and sensitive mice. Clin Exp Immunol 1990; 82:378-83.

19. Gottstein B, Hemphill A. Immunopathology of echinococcosis. In: Freedman DO, editor. Immunopathogenetic aspects of disease induced by helminth parasites. Basel: Karger Verlag, 1997; 66:177-208.

20. Siles LM, Felleisen RSJ, Hemphill A, Wilson W, Gottstein B. Stage-specific expression of the 14-3-3 gene in Echinococcus multilocularis. Mol Biochem 1998; 91:281-93.

21. Acs P, Szallasi Z, Kazanietz MG, Blumberg PM. Differential activation of PKC isozymes by 14-3-3 zeta protein. Biochem Biophys Res Com 1995; 216:103-9.

22. Morrison D. 14-3-3: modulators of signaling proteins? Science 1994; 226:56-7.

23. Gottstein B, Felleisen R. Protective immune mechanisms against the metacestode of Echinococcus multilocularis. Parasitol Today 1995; 11:320-6.

24. Magnani J, Steplewski Z, Koprowski H, Ginsburg V. Identification of the gastrointestinal and pancreatic cancer-associated antigen detected by monoclonal antibody 10-9 in the sera of patients as a mucin. Cancer Research 1983; 43:5489-92.

25. Khoo KH, Nieto A, Morris HR, Dell A. Structural characterisation of $\mathrm{N}$-glycans from Echinococcus granulosus hydatid cyst membrane and protoscolesces. Mol Biochem Parasitol 1997; 86:237-48.

Received 27 April 2001, accepted 5 May 2001

Corresponding author: A. Cerny, MD, Department of Medicine, Ospedale Civico, Via Tesserete 46, 6900 Lugano, Switzerland

Tel: +41-91-805 61 11, Fax: +41-91-805 6990 